\title{
Refuerzo virtual como estrategia - enseñanza, "GOCONQR" para potenciar el aprendizaje extra clase en estudiantes secundários
}

\section{Virtual reinforcement as a teaching - strategy, "GOCONQR" to enhance students' extra-class learning.}

DOI: $10.46932 / \mathrm{sfjdv2n2-169}$

Received in: March 1st, 2021

Accepted in: May 30th, 2021

\author{
Mauricio Xavier Prado Ortega \\ Magíster en Educación Superior \\ Current Institution: Universidad Técnica de Machala \\ Full address: Urbanización Santa Inés Mz F villa 18AB, Machala-Ecuador \\ E-mail:mprado@utmachala.edu.ec \\ Rosman José Paucar Córdova \\ Magíster en Interconectividad de Redes. \\ Current Institution: Universidad Técnica de Machala \\ Full address: Huaquillas-Ecuador \\ E-mail: rpaucar@utmachala.edu.ec \\ Anyeline Natalia González Segarra \\ Ingeniera de Sistemas - Docente Secundario. \\ Current Institution: Escuela Enriqueta de Wind de Laniado \\ Full address: Urbanización Santa Inés Mz F villa 18AB, Machala-Ecuador \\ E-mail: anyeline.gonzalez@educacion.gob.ec \\ Héctor Ramiro Carvajal Romero \\ Magíster en Administración de empresas \\ Current Institution: Universidad Técnica de Machala \\ Full address: Urbanización Santa Inés Mz F villa 23B, Machala-Ecuador \\ E-mail: hcarvajal@utmachala.edu.ec
}

\section{RESUMEN}

El constante fortalecimiento en la educación evidenciado por la incorporación de herramientas informáticas desarrolladas para apoyar el aprendizaje de los estudiantes y la adaptación de estrategias fáciles de usar con las que los docentes promueven y promueven la difusión de contenidos digitales promueven el interés por abordar el tema. de refuerzos virtuales como instrumento extra de clase, utilizando dispositivos móviles y portátiles. El presente estudio presenta una metodología con enfoque mixto que centra su análisis en la investigación acción asociada al abordaje diagnóstico de modelos pedagógicos en instituciones educativas de nivel medio para promover el uso de la tecnología a aquellas con la intervención de estudiantes universitarios de la Carrera de Pedagogía. de Ciencias Experimentales de la UTMACH a través de prácticas preprofesionales a través de un proyecto integrador de conocimientos. Entre los problemas más relevantes se encuentra la falta de motivación y desinterés de los estudiantes por los contenidos impartidos por los profesores en las asignaturas de ciencias blandas en el aula. Se utilizaron instrumentos como encuestas dirigidas a estudiantes de secundaria en estudio. Una solución alternativa presentada como objetivo general mediante la aplicación de GoConqr como herramienta tecnológica virtual complementaria a las clases de los alumnos con la ayuda de sus profesores 
de segundo bachillerato de la Escuela Kléber Franco Cruz. Entre los resultados más relevantes del estudio realizado cuando se utilizó GoConqr, se experimentaron algunos beneficios de su aplicación, que permitieron evaluar conocimientos, motivar la interacción y despertar el interés por los contenidos digitales incorporados en las asignaturas, incentivando el nivel de los estudiantes. de aprendizaje. La investigación concluye que el refuerzo virtual de contenidos presentados digitalmente mediante dispositivos móviles puede ser una estrategia docente y un elemento de acompañamiento para que el alumno mejore su interés por el aprendizaje.

Palabras clave: Tecnología Educativa, Aprendizaje Virtual, Docencia, Clase Extra

\begin{abstract}
The constant strengthening in education evidenced by the incorporation of computer tools developed to support student learning and the adaptation of easy-to-use strategies with which teachers promote and promote the dissemination of digital content promote interest in addressing the subject. of virtual reinforcements as an extra class instrument, using mobile and portable devices. The present study presents a methodology with a mixed approach that focuses its analysis on action research associated with the diagnostic approach of pedagogical models in medium-level educational institutions to promote the use of technology to those with the intervention of university students of the Career of Pedagogy of Experimental Sciences of the UTMACH through pre-professional practices through an integrative project of knowledge. Among the most relevant problems is the lack of motivation and disinterest of students for the content taught by teachers in soft science subjects in the classroom. Instruments such as surveys directed at secondary students under study were used. An alternative solution presented as a general objective through the application of GoConqr as a complementary virtual technological tool to the students' classes with the help of their teachers in the second baccalaureate of the Kléber Franco Cruz School. Among the most relevant results of the study carried out when GoConqr was used, some benefits of its application were experienced, which made it possible to assess knowledge, motivate interaction and awaken interest in the digital content incorporated into the subjects, encouraging the students' level of learning. The research concludes that the virtual reinforcement of content presented digitally using mobile devices can be a teaching strategy and an accompaniment element for the student to improve their learning interest.
\end{abstract}

Keywords: Educational Technology, Virtual Learning, Teaching, Extra Class

\title{
1 INTRODUCCIÓN
}

Durante el proceso de observación realizado en las aulas del Colegio Kléber Franco Cruz se determinaron diversos problemas que inciden en el proceso de aprendizaje de los estudiantes en relación a las asignaturas blandas como Biología, Literatura, Emprendimiento; mediante la observación determinaron problemas como el desinterés mostrado por la clase y desmotivación de los estudiantes hacia los contenidos tradicionales, por lo descrito se consideró como objetivo general del estudio incorporar la utilización del recurso tecnológico "GoConqr" para mejorar el aprendizaje de los estudiantes, en las asignaturas blandas en tres cursos de Segundo Bachillerato.

Entre los estudios publicados utilizando herramientas informáticas virtuales, uno de ellos hace mención y describe la reflexión que, como docentes, experimentamos todos los días un gran dilema: cómo 
desarrollar el programa de estudios de nuestras asignaturas de una manera funcional y atractiva frente al amplio contexto tecnológico en el que se insertan nuestros estudiantes. Sabemos que ese formato tradicional de clase ya no se ajusta a la realidad de nuestros estudiantes. La figura del maestro como único poseedor de conocimiento, cuya función es la transmisión del conocimiento a sus alumnos, debe deshacerse y reemplazarse por una perspectiva que lo considere un mediador (Sperandio, 2017).

La presente investigación tiene por finalidad identificar desde una aproximación diagnóstica experimental y la investigación acción hacia los diferentes problemas que puedan existir en el contexto educativo con la aplicabilidad de modelos pedagógicos contemporáneos, sobre todo en la actualidad con el aprendizaje de los estudiantes secundarios necesitan de la incorporación de la tecnología educativa. Además de la participación del docente y sus estrategias de enseñanza-aprendizaje al incorporar tecnología educativa sobre todo de manera virtual en el contexto extra clase para reforzar los contenidos impartidos en el aula. Con la incorporación de nuevas plataformas virtuales (LMS) se produjo un punto de inflexión por el número de herramientas que ofrecían, animando a los docentes a buscar sus posibles usos en la práctica, con la consecuencia de una mayor diversidad metodológica especialmente encontradas por los más innovadores, pues no siempre van unido o son consecuencias las nuevas posibilidades tecnológicas con nuevas prácticas (Cebrián de la Serna, 2011)

Actualmente hemos de enfrentarnos a nuevos retos ante los numerosos y diversos cambios surgidos en la sociedad. Y la educación tiene el cometido de educar a los sujetos en esas nuevas claves de la sociedad del conocimiento (Lobato \& Madinabeitia, 2011). Por tanto, es necesario que mediante la aplicación de un modelo pedagógico como el implementado por la UTMACH denominado desarrollador e integrador permiten y fomentan la realización de proyectos educativos, algunos autores promueven que la actual visión de la educación para el nuevo siglo, implica cambios en el quehacer de los docentes y en su formación inicial y continua. Atendiendo a los retos de la sociedad del conocimiento a los que la educación invita a revisar con profundidad su esencia, en la cual el centro de la misma es el ser humano y su evolución, surge el interés por analizar el impacto de éste en el aprendizaje de los estudiantes (Moreno et al., 2014).

Dado estos puntos negativos identificados en el alumnado mediante un proyecto integrador de saberes por parte de los estudiantes de la carrera de Ciencias experimentales de la UTMACH motivó el uso de una App como GoConqr con el propósito de mejorar la atención del estudiante, en la cual a través de clases experimentales con un buen manejo de la herramienta explicada en el aula y manipulada en los hogares como refuerzo virtual propició la realización de pequeños test y evaluaciones de tipo opción múltiple de las que se obtuvieron resultados óptimos, los cuales prometen a futuro la aplicabilidad de GoConqr y otras más en el aprendizaje de los educandos. 
A continuación, se describen aspectos teóricos que evidencian a profundidad el enfoque bajo aspectos como la motivación, interés el estilo pedagógico, por lo que abre una línea de investigación y experimentación en Tecnología Educativa;

\section{MOTIVACIÓN DEL ESTUDIANTE POR EL APRENDIZAJE.}

Para algunos expertos en desarrollo infantil expresan que se debe contribuir a que los alumnos se sientan motivados para aprender implica la existencia en ellos de total claridad y coherencia en cuanto al objetivo del proceso de aprendizaje, que lo encuentren interesante y que se sientan competentes para resolver el reto (Ospina Rodríguez, 2006).

Por lo tanto, es necesario examinar dos tipos de motivaciones: La motivación intrínseca se puede definir como aquella que procede del propio sujeto, que está bajo su control y tiene capacidad para autoreforzarse. Se supone que cuando se disfruta ejecutando una tarea se induce una motivación intrínseca positiva. En segundo lugar, puede aparecer una motivación extrínseca negativa opuesta a la positiva que conduce

a la no ejecución de la tarea (conducta de evitación) porque está vinculada con experiencias pasadas negativas. Por lo tanto, además de impedir la motivación intrínseca positiva, las emociones negativas también producen motivación intrínseca negativa. Una de las emociones negativas que conlleva a la no ejecución o evitación es el "aburrimiento" (Anaya-Durand \& Anaya-Huertas, 2010).

Este nuevo enfoque hace que se requiera un nuevo rol para la figura del profesor pues deja de ser por un lado el actor central y por otro sus actividades cambian de ser directivas a ser orientadoras y mediadoras, cuya función principal cambia de ser instructora a ser promotora de ambientes de aprendizaje. En este sentido, el trabajo colaborativo exige que los miembros del grupo compartan las tareas y las aportaciones para un objetivo en común (Rodríguez Zamora \& Espinoza Núñez, 2017).

\section{MEJORAR O DESPERTAR EL INTERÉS DEL ESTUDIANTE POR EL APRENDIZAJE.}

Definitivamente al estudiante le agrada lo nuevo y que tenga retos. Es importante entonces explorar la fusión de los conceptos red social y Entorno Virtual de Aprendizaje (EVA), da lugar a un nuevo escenario en donde profesores y estudiantes encuentren el espacio necesario para interactuar de manera informal en un ambiente de aprendizaje formal, dando como resultado un modelo de aprendizaje colaborativo y social (Carlos Torres-Diaz et al., 2013).

Es importante establecer que ninguna persona aprende desde la misma manera, ni existe una institución que lo conozco todo en materia de enseñanza. Hablamos de cada institución como diferente, dónde los cambios a veces no surten los efectos deseados y encuentran resistencias y por ello deben de 
planificarse meticulosamente y dónde es preciso comprometer en el trabajo colaborativo y constructivo a todos los componentes por diferentes vías de actuación motivación (Trujillo Torres et al., 2011).

De ahí que los docentes y estudiantes deban desarrollar habilidades para responder a las demandas de la sociedad del conocimiento, y sean capaces de procesar y aplicar críticamente la información relevante y pertinente en la resolución de problemas de manera creativa e innovadora. Para responder a este reto, muchas instituciones educativas han adoptado nuevos entornos de aprendizaje que facilitan un aprendizaje flexible y continuo e incorporan la utilización de las herramientas de trabajo colaborativo en red (Martínez \& Duart, 2016).

\section{ESTILOS Y MODELOS PEDAGÓGICOS CONTEMPORÁNEOS.}

No existe un modelo pedagógico único, omnipotente, capaz de solucionar todos los problemas de aprendizaje que tienen los estudiantes, y que permita agrupar la amplia variedad de tipologías que hayan proliferado en la historia de la educación y que se han nutrido de los avances de la Psicología y de las teorías del aprendizaje. Piaget no era pedagogo, Vigotsky tampoco. Ambos eminentes investigadores desde la Psicología han hecho invaluables aportes a la educación del ser humano (Ocaña, 2013).

Por modelo también se entiende algo que media entre los aspectos característicos de una teoría y la praxis. Ampliando la conceptualización a partir de esto, puede afirmarse que un modelo es una herramienta que se utiliza para describir, decidir e interpretar actitudes en diferentes situaciones, haciendo viable el reproducir hipótesis y

elaborar estrategias para su intervención (Garzón-Saladen \& Romero-González, 2018).

De las concepciones anteriormente expuestas surge el concepto de aprendizaje según Piaget: “El aprendizaje se produce a lo largo de un proceso en el que el sujeto, a través de la experiencia, dentro de un contexto real, mediante la manipulación de objetos y la interacción con las personas, genera o construye conocimiento, modificando, en forma activa, sus esquemas cognoscitivos, empleando sus propios esquemas para la asimilación de la realidad que proviene de la actividad constructiva de la inteligencia del sujeto" (Fonseca \& Bencomo, 2011).

En pleno siglo XX se originó el modelo desarrollista o cognoscitivismo. De esa manera este modelo se encuentra orientado por la educabilidad, al estudiante le sobreviene la posibilidad de autorealizarse enteramente, es decir, que contaría con la disposición para la autoformación de su personalidad a partir de sus propias potencialidades. La educación, vista de este modelo, se potencializa hacia las múltiples posibilidades como una promesa a cerca de lo cual el ser humano puede imaginarse a partir de la autonomía de aprendizaje y de la autogestión (Cantor Isaza \& Altavaz Ávila, 2019). 
En la actualidad por medio de la virtualidad el modelo de formación en competencias implica un proceso de cambio que incide directamente en la visión y pensamiento del estudiante, fomentando el pensamiento crítico y su inteligencia práctica (Belmonte \& Bernárdez-Gomez, 2020).

\section{TENDENCIAS DE LA TECNOLOGÍA EDUCATIVA.}

Como un aporte a la educación es necesario describir algunos aportes de este siglo entre lo que se destacan: cambios en los discursos; apertura a las posibilidades de las TIC como medio para comunicarse, para conocer otras personas, para buscar información y trabajo, y para estudiar conciliando vida laboral y familiar e incluso para desarrollar nuevos hábitos; y adquisición de equipos informáticos e instalación de Internet en los domicilios (Fernández Sánchez \& Valverde Berrocoso, 2014).

Entre las nuevas tendencias para la armonía de la educación y la tecnología tenemos el concepto de aprendizaje electrónico o e-learning ha tomado fuerza en las últimas décadas, las aulas virtuales de elearning se han constituido en poderosas herramientas a través de las cuales se ha incrementado la calidad y cantidad de los procesos formativos (Ladaysé Mayorga-Albán et al., 2020).

$\mathrm{Al}$ incorporar tecnología a la educación es necesario considerar que, en el diseño de actividades didácticas, es posible salir de lo clásico e innovar con la incorporación de entornos digitales, sacando el máximo potencial de las nuevas tecnologías mediante el diseño de cuestionarios y obtención de resultados más rápidos. Bajo este prisma, el diseño de una actividad didáctica puede ser realizado mediante una actividad virtual donde se puede trabajar con más variables que del modo clásico. Con el uso de entornos virtuales no sólo puede ayudar en el afianzamiento de los conocimientos recién aprendidos sino, tal y como hemos expresado en apartados anteriores, su desarrollo y asimilación (Caraballo et al., 2017).

\section{MATERIALES Y MÉTODOS.}

Para el presente estudio se realizó un proyecto integrador de saberes en el ámbito educativo, mediante una experimentación y la investigación acción, que contó con la participación de cinco estudiantes de la UTMACH de la Carrera de Pedagogía de Ciencias Experimentales. La experiencia con el desarrollo y aplicación de esta estrategia muestra que como herramienta pedagógica que familiariza al estudiante con la investigación, su naturaleza y sus fases. Favorece el desarrollo de la autonomía, el pensamiento crítico, la comunicación oral y escrita, la capacidad de análisis, y, de habilidades metacognitivas como la planeación, la argumentación, la solución de problemas y la toma de decisiones entre otras. Además, permite la adquisición de habilidades y competencias para apoyar los conocimientos teóricos, prácticos y técnicos necesarios para lograr un ejercicio cualificado de su actividad profesional (Castillejo Olán et al., 2017) 
En una primera fase, la institución secundaria de acogida permitió desarrollar la implementación del proyecto mediante las prácticas preprofesionales con clases experimentales por dos meses en el periodo académico 2019-2020, bajo la tutela y participación de cuatro docentes del colegio y cuatro cursos de segundo de bachillerato aplicando GOCONQR como una herramienta que permita reforzar virtualmente de forma extra clase contenidos que se pueden desarrollar en la clase presencial. El número total de estudiantes secundarios fue de 120 participantes. Como se puede visualizar el Tabla 1.

\begin{tabular}{|c|c|c|c|c|}
\hline Sexo & Edad & $\begin{array}{l}\text { Bachillerato } \\
\text { Técnico }\end{array}$ & $\begin{array}{l}\text { General } \\
\text { Unificado }\end{array}$ & Total \\
\hline \multirow{5}{*}{ Masculino } & 14 & 4 & 2 & 6 \\
\hline & 15 & 30 & 17 & 47 \\
\hline & 16 & 1 & 5 & 6 \\
\hline & 17 & 0 & 1 & 1 \\
\hline & Subtotal & 35 & 25 & 60 \\
\hline \multirow[t]{6}{*}{ Femenino } & 14 & 0 & 5 & 5 \\
\hline & 15 & 18 & 23 & 41 \\
\hline & 16 & 4 & 8 & 12 \\
\hline & 17 & 2 & 0 & 2 \\
\hline & Subtotal & 24 & 36 & 60 \\
\hline & Total & 59 & 61 & 120 \\
\hline
\end{tabular}

En una segunda fase mediante la investigación - acción se aplicó un enfoque mixto, que supone entender la enseñanza como un proceso de investigación y de continua búsqueda. Conlleva entender el oficio docente, integrando la reflexión y el trabajo intelectual en el análisis de las experiencias que se realizan, como un elemento esencial de lo que constituye la propia actividad educativa. Entre los instrumentos se establecieron dos cuestionarios uno aplicado durante la etapa de observación y otra luego de las clases experimentales utilizando la Herramienta GOCONQR. Se desarrollaron preguntas de opción múltiple, mediante una escala Likert. Las preguntas se organizaron en categorías para identificar los escenarios en función de la utilización de tecnología educativa.

En una tercera fase se realizaron clases experimentales en las aulas en compañía de los docentes de la institución educativa en conjunto con la intervención de los estudiantes de la UTMACH que realizaban las prácticas preprofesionales, en la cual se explicaba los contenidos digitales que los estudiantes secundarios del segundo bachillerato debían abordar en sus hogares de manera virtual como refuerzo extra clase en los cuatro cursos intervenidos. El proceso de análisis de datos estadísticos presentados en las tablas, fueron almacenados y tabulados mediante el programa SPSS IBM versión 22 para sistema operativo Windows de 64 bits. 
En la cuarta fase se expusieron los resultados ante las autoridades del Colegio sobre la utilización de interactuar con tecnología educativa para reforzar los conocimientos de manera extra clase mediante la herramienta de uso gratuito GOCONQR como alternativa de aula virtual, y así evidenciar el estudio en consecuencia a los objetivos planteados por la investigación y la socialización de los beneficios para las instituciones, tanto la interventora como la intervenida.

\section{RESULTADOS Y DISCUSIONES}

Entre los resultados más significativos, se identificó que una de las causas que restringe la utilización de herramientas tecnológicas en los establecimientos educativos secundarios físcales es la ausencia del servicio de internet en las aulas que propicie su interacción; esto sumado a la prohibición de utilizar dispositivos como celulares en clases. Por tanto, al indagar sobre el acceso a internet en los hogares de los estudiantes, manifiestan que cuentan con la conexión del servicio de diversas maneras como planes con proveedores locales, y acceso desde dispositivos móviles mediante consumo de datos. De esta manera fue factible enviar las tareas extra clases que solo requerían de máximo de tiempo de 30 minutos y aplicar GOCONQR para su utilización virtual. Como se indica en la figura 1.

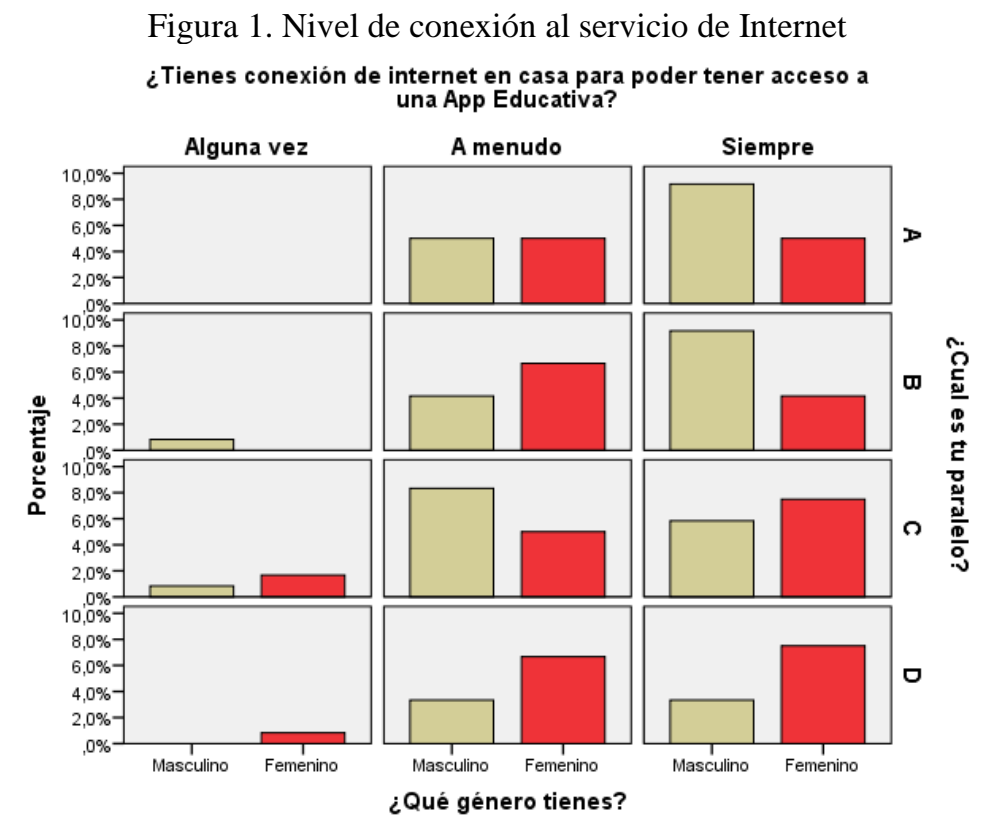

Fuente: Estudiantes del Colegio de Bachillerato Kléber Franco Cruz.

Además, se identificó que los estudiantes tienden a realizar sus tareas e incrementan su cumplimiento cuando interactúan con la tecnología educativa. Utilizando la herramienta GoConqr, que es un entorno de estudio creado por un docente de manera personalizada, online y gratuito. Ayuda y promueve el aprendizaje de los estudiantes mediante clases interactivas a las que pudieron acceder 
virtualmente por medio de computadoras y dispositivos móviles mejorando y reforzando los contenidos dictados en el salón de clases. Ver tabla 2.

Tabla 2. Nivel de Participación con GOCONQR

\begin{tabular}{lllll}
\hline \multicolumn{5}{c}{ ¿Participaste en actividades extra clases con GOCONQR? } \\
Alguna vez & A menudo & Siempre & Total \\
$\begin{array}{l}\text { Bachillerato } \\
\text { Técnico }\end{array}$ & 14 & 29 & 16 & 59 \\
General Unificado & 12 & 28 & 21 & 61 \\
\hline Total & 26 & 57 & 37 & 120 \\
\hline
\end{tabular}

El ambiente de GoConqr tiene una interfaz que favorece la interacción con los estudiantes y es muy útil para un docente, él envió de tareas mejoró notablemente de igual manera la participación de los estudiantes utilizando dispositivos móviles como alternativa de conectividad. Ver tabla 3.

Tabla 3. Realizar tareas con el uso de Goconqr

\begin{tabular}{llllll}
\hline \multicolumn{5}{c}{ Según su criterio ¿es interesante realizar tareas con el uso de Goconqr? } \\
\hline & Nunca & Alguna vez & A menudo & Siempre & Total \\
Bachillerato & 3 & 9 & 27 & 20 & 59 \\
$\begin{array}{l}\text { Técnico } \\
\text { General Unificado }\end{array}$ & 0 & 5 & 25 & 31 & 61 \\
\hline Total & 3 & 14 & 52 & 51 & 120 \\
\hline
\end{tabular}

Al revisar y contrastar los aprendizajes de los contenidos más el aporte del refuerzo virtual, los resultados fueron los esperados en los estudiantes, una mayor percepción, participación e interés por las asignaturas de una manera autónoma.

Figura 2. Clases experimentales para evaluar el refuerzo virtual

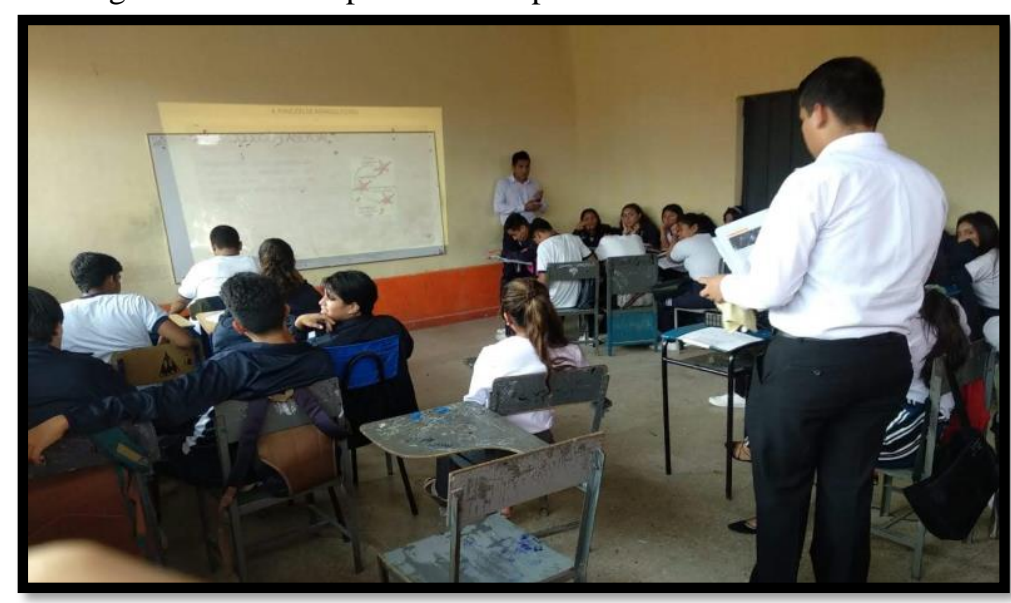

Fuente: Estudiantes del Colegio de Bachillerato Kléber Franco Cruz. 


\section{CONCLUSIONES}

Promover la utilización de herramientas tecnológicas como una inducción al aprendizaje virtual y colaborativo con la interacción de la plataforma GoConqr, se evidenció en los estudiantes un incremento de su participación en el aula de clases, mediante el aporte de clases experimentales y el refuerzo virtual.

Como refuerzo virtual extra clase con la ayuda de la plataforma GoConqr, se evidencia que los estudiantes en sus hogares se interesaron por sus tareas de manera entretenida mejorando su dinámica de aprendizaje.

Otra característica de la presente investigación es que mediante GoConqr, se evidenció que el docente puede optar por metodologías innovadoras y otros puntos de vista al momento de realizar un refuerzo extra clase virtual utilizando dispositivos móviles.

Entre las limitaciones debemos considerar un aspecto como el acceso a internet en el sector educativo y consecuentemente con la intermitencia y disponibilidad de este servicio en los domicilios de los estudiantes impedirían su implementación. Es emergente que los docentes particularmente deben interesarse por el manejo de plataformas, aplicaciones y herramientas virtuales que les permitan apropiarse de conocimiento para elaborar contenidos digitales e interactivos en correspondencia al entorno educativo actual y desarrollo tecnológico en el que nos desenvolvemos y que los estudiantes experimentan. 


\section{REFERENCIAS}

Anaya-Durand, A., \& Anaya-Huertas, C. (2010). ¿Motivar para aprobar o para aprender? Estrategias de motivación del aprendizaje para los estudiantes. Tecnol. Ciencia Ed. (IMIQ), 25(1), 5-14. https://www.redalyc.org/articulo.oa?id=48215094002

Belmonte, M. L., \& Bernárdez-Gomez, A. (2020). Perfiles profesionales del pedagogo. South Florida Journal of Developmen, $305-319$. https://southfloridapublishing.com/ojs/index.php/jdev/article/view/68/82

Cantor Isaza, J. F., \& Altavaz Ávila, A. C. (2019). Los modelos pedagógicos contemporáneos y su influencia en el modo de actuación profesional pedagógico. Varona. Revista Científico Metodológica, versión impresa ISSN 0864-196X, 68, 1-6. http://scielo.sld.cu/scielo.php?script=sci_arttext\&pid=S199282382019000100019

Caraballo, M., Es Herranz Peinado, A. M. A., Es, P. P., González, S., Manuela, M., \& Es, M. (2017). Gamificación en la educación, una aplicación práctica con la plataforma Kahoot. Anales de ASEPUMA, $\begin{array}{llllll}\text { ISSN-e } & 2171-892 X, & N^{\circ} . & 25, & 2017, & 25,\end{array}$ https://dialnet.unirioja.es/servlet/articulo?codigo=6210181\&info=resumen\&idioma=ENG

Carlos Torres-Diaz, J., Inés Jara, D., \& Valdiviezo, P. (2013). Integración de redes sociales y entornos virtuales de aprendizaje Integrating Social Networks and Virtual Learning Environments. Revista de Educación a Distancia (RED), 35, 1-8. http://www.um.es/ead/red/35

Castillejo Olán, R., Rodríguez Torres, Á., Páez Granja, R., Altamirano Vaca, E., \& Granados Romero, J. (2017). El Proyecto Integrador de Saberes. Análisis crítico desde la perspectiva de alumnos y docentes (revisión). Olimpia: Publicación científica de la facultad de cultura física de la Universidad de Granma, 14(46), 99-110. https://revistas.udg.co.cu/index.php/olimpia/article/view/189

Cebrián de la Serna, M. (2011). Los ePortafolios en la supervisión del Practicum: Modelos pedagógicos y soportes tecnológicos. DIGIBUG Principal, Profesorado. Revista de Curriculum y Formación del Profesorado , 15, 91-107. https://digibug.ugr.es/handle/10481/15361

Fernández Sánchez, M. R., \& Valverde Berrocoso, J. (2014). Comunidades de práctica: un modelo de intervención desde el aprendizaje colaborativo en entornos virtuales - Dialnet. Comunicar: Revista científica iberoamericana de comunicación y educación, 42, 95-105. https://doi.org/http://dx.doi.org/10.3916/C42-2014-09

Fonseca, H., \& Bencomo, M. (2011). Teorías del aprendizaje y modelos educativos: revisión histórica. Salud, Arte y Cuidado, 4(1), 71-93. https://dialnet.unirioja.es/servlet/articulo?codigo=3938580

Garzón-Saladen, Á., \& Romero-González, Z. (2018). Los modelos pedagógicos y su relación con las concepciones del derecho: puntos de encuentro con la educación en derecho. REVISTA DE INVESTIGACIÓN, DESARROLLO E INNOVACIÓN, https://doi.org/10.19053/20278306.v8.n2.2018.7968

Ladaysé Mayorga-Albán, A. I., Enrique Aveiga-Paini, C. I., \& Raúl Fierro-Saltos III, W. (2020). Los modelos e-learning en el desarrollo del aprendizaje colaborativo en la educación superior. Dominio de las Ciencias, ISSN-e 2477-8818, Vol. 6, No. 2, 2020 (Ejemplar dedicado a: Vol 6, No 2 (2020): Abril-Junio), págs. 847-865, 6(2), 847-865. https://doi.org/10.23857/dc.v6i2.1198 
Lobato, C., \& Madinabeitia, A. (2011). Perfiles Motivacionales del Profesorado ante la Formación en Metodologías Activas en la Universidad. Formación universitaria, 4(1), 37-48. https://doi.org/10.4067/s0718-50062011000100006

Martínez, R. A., \& Duart, J. M. (2016). Nuevas tendencias de aprendizaje colaborativo en e-learning. Claves para su implementación efectiva. Estudios Pedagogicos, 42(1), 271-282. https://doi.org/10.4067/S0718-07052016000100017

Moreno, C. P., Molina, Y. A., \& Chacón, J. A. (2014). Impacto del estilo pedagógico integrador en los estudiantes de Licenciatura en educación Básica de la Facultad de Estudios a distancia. Formacion Universitaria, 7(6), 37-44. https://doi.org/10.4067/S0718-50062014000600005

Ocaña, A. O. (2013). Hacia una nueva clasificación de los modelos pedagógicos: el pensamiento configuracional como paradigma científico y educativo del siglo xxi. Praxis, 7(1), 121-137. https://doi.org/10.21676/23897856.18

Ospina Rodríguez, J. (2006). La motivación, motor del aprendizaje | Revista Ciencias de la Salud. Revistas Ciencias de la Salud, 4, 158-160. https://doi.org/http://dx.doi.org/10.12804/revsalud

Rodríguez Zamora, R., \& Espinoza Núñez, L. A. (2017). Trabajo colaborativo y estrategias de aprendizaje en entornos virtuales en jóvenes universitarios. RIDE Revista Iberoamericana para la Investigación y el Desarrollo Educativo, 7(14), 103. https://doi.org/10.23913/ride.v7i14.274

Sperandio, N. E. (2017). Anais do Encontro Virtual de Documentação em Software Livre e Congresso Internacional de Linguagem e Tecnologia Online. Anais do Encontro Virtual de Documentação em Software Livre e Congresso Internacional de Linguagem e Tecnologia Online, 6(1), 1-6. http://www.periodicos.letras.ufmg.br/index.php/anais_linguagem_tecnologia/article/view/12130/10337

Trujillo Torres, J. M., Cáceres Reche, M. P., Hinojo Lucena, F. J., \& Aznar Díaz, I. (2011). Aprendizaje cooperativo en entornos virtuales. El proyecto Redes Educativas y Organizativas Interuniversitarias. Educar, 47(1), 95-119. https://doi.org/https://doi.org/10.5565/rev/educar.73 